Submitted to the Editor of the ApJ Letters on December 7, 1997

\title{
Proper Motions of Ionized Gas at the Galactic Center: Evidence for Unbound Orbiting Gas
}

\author{
F. Yusef-Zadeh \\ Department of Physics and Astronomy, Northwestern University, Evanston, IL 60208 \\ (zadeh@nwu.edu) \\ D. A. Roberts \\ NCSA, 1002 W. Green St., Urbana, IL 61801 (dougr@ncsa.uiuc.edu) \\ J. Biretta \\ STScI, 3700 San Martin Drive, Baltimore, MD 21218 (biretta@stsci.edu)
}

Received 00 December 1997; accepted 001996 


\begin{abstract}
We present radio continuum observations of the spiral-shaped ionized feature (Sgr A West) within the inner pc of the Galactic center at three epochs spanning 1986 to 1995 . The VLA A-configuration was used at $\lambda 2 \mathrm{~cm}$ (resolution of $\left.0 \prime 2 \times 00^{\prime \prime} 2\right)$. We detect proper motions of a number of features in the Northern and Eastern Arms of Sgr A West including the ionized gas associated with IRS 13 with $\mathrm{V}(\mathrm{RA})=113 \pm 10, \mathrm{~V}($ Dec $)=150 \pm 15 \mathrm{~km} \mathrm{~s}^{-1}$, IRS 2 with $\mathrm{V}(\mathrm{RA})=$ $122 \pm 11, \mathrm{~V}(\mathrm{Dec})=24 \pm 34 \mathrm{~km} \mathrm{~s}^{-1}$ and the Norther Arm V(RA) $=126 \pm 30$, $\mathrm{V}($ Dec $)=-207 \pm 58 \mathrm{~km} \mathrm{~s}^{-1}$. We also report the detection of features having transverse velocities $>1000 \mathrm{kms}^{-1}$ including a head-tail radio structure, the "Bullet", $\approx 4$ " northwest of Sgr A* with $\mathrm{V}(\mathrm{RA})=722 \pm 156, \mathrm{~V}(\mathrm{Dec})=832 \pm 203$ $\mathrm{km} \mathrm{s}^{-1}$, exceeding the escape velocity at the Galactic center.

The proper motion measurements when combined with previous H92 $\alpha$ radio recombination line data suggest an unambiguous direction of the flow of ionized gas orbiting the Galactic center. The measured velocity distribution suggests that the ionized gas in the Northern Arm is not bound to the Galactic center assuming a 2.5 million solar mass of dark matter residing at the Galactic center. This implies that the stellar and ionized gas systems are not dynamically coupled, thus, supporting a picture in which the gas features in the Northern Arm and its extensions are the result of an energetic phenomenon that has externally driven a cloud of gas cloud into the Galactic center.
\end{abstract}

Subject headings: galaxies: ISM - Galaxy: center - ISM: individual (Sgr A East and Sgr A West) — ISM: magnetic fields 


\section{Introduction}

The ionized gas known as Sagittarius A West (Sgr A West) appears as a three-Arm spiral-like structure (North, East, and West Arms) engulfing the inner pc of the Galaxy where Sgr A*, the compact radio source at or near the dynamical center of the Galaxy lies (Ekers et al. 1983). These features are surrounded by neutral gas in the circumnuclear disk (CND) rotating with the velocity of about $100 \mathrm{kms}^{-1}$ at the distance of 2 pc from the Galactic center (e.g. Güsten et al. 1988). The kinematics of ionized gas surrounding Sgr A* show systematic velocities along various components of Sgr A West including Western Arc with a radial velocity structure which varies regularly between -100 and $+100 \mathrm{kms}^{-1}$ in the North-South direction (e.g. Serabyn et al. 1988; Herbst et al. 1993; Roberts \& Goss 1993). However, the velocity structure of the inner $10^{\prime \prime}$ where there is a hole in the distribution of ionized gas, known as the "mini-cavity", becomes increasing more negative $\approx-350 \mathrm{kms}^{-1}$ approaching Sgr A* (Yusef-Zadeh, Morris \& Ekers 1989; Roberts, Yusef-Zadeh \& Goss 1996, hereafter RYG).

Recent observations of stellar proper motions shows evidence of a $2.5 \times 10^{6} M_{\odot}$ object lying close to the position of Sgr A* (Eckart and Genzel 1997). The stars orbit randomly around the Galactic center with increasing velocity dispersion around Sgr A*, reflecting the gravitational potential of central mass. The ionized gas, on the other hand, is part of a coherent flow with systematic motion that is decoupled from the stellar orbits. Understanding the kinematics of the system of ionized gas is complicated by its incomplete view of the 3-dimensional geometry with respect to $\operatorname{Sgr} \mathrm{A}^{*}$ as well as by the interaction of orbiting gas with non-gravitational forces, such as the winds from the cluster of hot mass-losing stars near the Galactic center. To examine the gas kinematics and the true geometry of the ionized flow, in this Letter we present the results of proper motion measurements of ionized gas at $\lambda 2 \mathrm{~cm}$. 


\section{Observations}

Radio continuum observations were carried out with the Very Large Array of the National Radio Astronomy Observatory" in 1986.167, 1990.375 and 1995.557 at $\lambda 2 \mathrm{~cm}$ in the A-configuration using similar $u v$ coverage, identical bandwidths of $12.5 \mathrm{MHz}$ and identical phase center at $\operatorname{RA}(1950)=17^{\mathrm{h}} 42^{\mathrm{m}} 29^{\mathrm{s}} .33$, $\operatorname{Dec}(1950)=-29^{\circ} 59^{\prime} 17^{\prime \prime} .0$. We have employed a technique in which the variability of $\mathrm{Sgr} \mathrm{A}^{*}$ is removed on every ten-minute scan to improve the dynamic range of radio continuum images. In order to minimize the difference in calibration errors between three different data sets, they were cross calibrated against the 1990.375 epoch before the final images were constructed. To measure the two-dimensional transverse velocities with respect to $\operatorname{Sgr} \mathrm{A}^{*}$, we generated Maximum Entropy images, and then applied cross-correlation method of Biretta, Owen, \& Cornwell (1989) This method which has been exhaustively tested (Biretta et al. 1995) determines the fractional pixel position shift between images (pixel size $35 \times 35$ milli-arcseconds) which maximizes their cross-correlation. Uncertainties on the velocities were estimated by extracting 20 noise images from a CLEAN image in regions beyond the inner $60^{\prime \prime}$ of the Galactic center, adding these to the MEM images, and repeating the cross correlations; position uncertainties, and hence velocity uncertainties, were determined from the dispersion in the results for these 20 noise images. The rms noises of 1986, 1990 and 1995 images are 0.13, 0.104 and 0.16 mJy beam $^{-1}$, respectively.

Sgr $A^{*}$ serves as an excellent reference point because of its small proper motion: $\mathrm{V}(\mathrm{RA})=-13 \pm 13, \mathrm{~V}(\mathrm{Dec})=-11 \pm 9 \mathrm{kms}^{-1}$ (Backer 1996), and is likely to be directly associated with the compact, massive object. The above technique was applied to two bright radio continuum sources surrounding IRS 13 and IRS 10 continuum sources The

\footnotetext{
${ }^{1}$ The National Radio Astronomy Observatory is a facility of the National Science Foundation, operated under a cooperative agreement by Associated Universities, Inc.
} 
uncertainties from our data for the east-west and north-south components of motion are 11

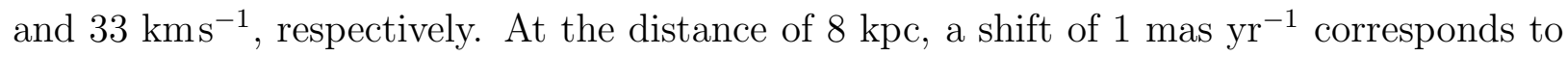
about $39 \mathrm{kms}^{-1}$. The velocity uncertainty in the radial direction is much smaller than in transverse directions.

\section{Results}

Figure 1a shows the pseudo-color representation of the continuum image at the resolution of 0 .' $1 \times 00^{\prime \prime} 2$ with 22 boxes superposed. Boxes are shown where proper motion of ionized gas is detected above $3 \sigma$ level, except Box 21, in at least one direction ( $\alpha$ or $\delta$ ). Boxes 21 and 22 which surround IRS 1 and IRS 8, respectively, show transverse velocities at $<3 \sigma$ level, but nonetheless they are important because of the low upper limits to their velocities. Table 1 shows the transverse motions of ionized gas averaged over the size of each box (column 5) in $\alpha$ and $\delta$ (columns 6 and 7). The radial velocities averaged over each box based on radio recombination line measurements (RYG) is shown in column 8 . The radial velocities of the features whose continuum emission is too weak for H92 $\alpha$ line detection or too compact are not reported. The angular distance of the center of each box with respect to $\operatorname{Sgr} \mathrm{A}^{*}$ is shown in radial, $\alpha$, and $\delta$ directions in columns 2,3 and 4, respectively. The total measured velocities and the upper limits to the corresponding escape velocities of ionized gas (determined using the projected distance (r) from Sgr A* with a mass of $\left.2.5 \times 10^{6} M_{\odot}\right)$ are shown in the last two columns.

Figure $1 \mathrm{~b}$ shows contours of $\lambda 2 \mathrm{~cm}$ continuum image from A-array observations at $0 \prime \prime 1 \times 0$ '. 2 resolution superposed on the pseudo-color representation of the radial velocity distribution of $\mathrm{H} 92 \alpha$ recombination line emission with spectral and spatial resolutions of $14 \mathrm{kms}^{-1}$ and $0^{\prime \prime} 75 \times 1$.'2 (RYG), respectively. The vectors drawn from the center of each box show the orientation of the transverse velocities and their lengths represent their 
magnitude. The regions with the highest signal-to-noise ratio $(>10)$ averaged roughly over 1.5 square arcseconds are shown as Box 6 and 10 in Table 1 where the continuum emission is strongest. The plots in Figure 2 show the change in $\alpha$ and $\delta$ of the IRS 13 (Box 6) source as a function of three epochs. Proper motions were determined by a least-squared fit to the data where the position values were weighted by their uncertainties.

The western half of the mini-cavity is represented by Boxes 6, 8, 10 and 11 where the prominent clusters of hot stars, IRS 13 and IRS 2 are embedded within extended ionized gas. From [NeII] and H92 $\alpha$ line observations the ionized gas in this region shows high radial velocities $\approx-300 \mathrm{kms}^{-1}$. The transverse velocities vary from 24 and $150 \mathrm{kms}^{-1}$, which are much less than their radial velocity components. The filamentary ionized structure connecting the IRS 13 and IRS 2 (Box 11) features also shows transverse velocities directed toward northwest.

The eastern half of the mini-cavity is represented by Boxes 1, 2 and 5. Box 4 corresponds to the radio Blob $\zeta$ (Yusef-Zadeh, Morris \& Ekers 1990; Zhao et al. 1991) lying near the center of the mini-cavity. Again, all the features to the east and near the center of the mini-cavity appear to have transverse velocities predominantly directed to the west with the exception of the anomalous velocity feature in Box 1 with transverse velocities exceeding $1000 \mathrm{kms}^{-1}$. The trend of westerly transverse motion of the ionized gas is also seen along the Northern and Eastern Arms (Box 3, 12, 15, 16, 17, 18, 19).

Figure 1b also displays an anomalous high-velocity feature in Sgr A West. This source called the "Bullet", as seen in Box 9, lies about 4" northwest of Sgr A* and shows a total transverse velocity of $1100 \mathrm{kms}^{-1}$ which is much greater than the upper limit to the escape velocity of $373 \mathrm{kms}^{-1}$ at its projected distance from Sgr A* (see Table 1). The low-resolution H92 $\alpha$ measurements of this region (Roberts \& Goss 1993) obtained using a limited velocity coverage $\left(-200 \mathrm{kms}^{-1}<V_{\mathrm{LSR}}<+200 \mathrm{kms}^{-1}\right)$ show extended line emission 
with radial velocities of about $-36 \mathrm{kms}^{-1}$ which is not likely associated with the Bullet. The peak flux density at $\lambda 1.2 \mathrm{~cm}$ is $\approx 2.5 \mathrm{mJy}$ within a beam of $0^{\prime \prime} .31 \times 00^{\prime \prime} .22$. Assuming that the Bullet is an optically thin thermal source at the temperature of $10^{4} \mathrm{~K}$, the electron density would be $3.6 \times 10^{4} \mathrm{~cm}^{-3}$, which is similar to typical electron densities of Blobs (Wardle \& Yusef-Zadeh 1991). The mass of the Bullet would be $8 \times 10^{-4} M_{\odot}$ assuming a spherical size of radius $6 \times 10^{-3} \mathrm{pc}$.

The proper motion velocity of the Bullet derived from the image correlation technique is large enough that the positions of the peak are displaced by a significant fraction of the synthesized beam on the plane of the sky between the various epochs. The velocity determined from measuring the displacement of the peak agrees with that determined by the correlation method. Figure 3 shows the transverse motion of the peak emission in the three different epochs superposed on a greyscale image of the Bullet (including the the tail) from the 1990 observations. The most recent image (1995) shows the peak to be at the position of the head of the source, with the tail pointing roughly toward the head in the earlier observations. The images shown in Figure 3 provide confidence in the results obtained from two completely different techniques of proper motion measurements.

\section{Discussion}

By combining the transverse and radial velocities, we are able to unambiguously determine the direction of ionized flow at the Galactic center. The predominant component of the motion in the plane of the sky is from east to west for most of the measured features with the exception of few places where the velocity of ionized gas is anomalously large. It appears that the flow of ionized gas in the Northern Arm (Box 12) originates in the northeast with red-shifted velocities in the orbital plane. The ionized gas then follows an orbital trajectory to the southwest as it crosses the plane of the sky and passes by Sgr A* 
before the ionized gas moves to the northwest.

One idea that has been suggested to explain the origin of the mini-cavity and its unusual characteristics (e.g. Lutz et al. 1993; Melia et al. 1996) is the the collision of fast-moving Blobs with the orbiting ionized gas. The Blobs are hypothesized to be formed as a result of high velocity winds of IRS 16 cluster escaping from but focussed by the gravitational potential of Sgr A*. This focusing mechanism allows the diffuse outflowing materials to collide with each other and form dense Blobs of ionized gas leaving the gravitational potential of Sgr A* (Wardle \& Yusef-Zadeh 1992). The anomalous high-velocity features seen in Boxes 1, 4, 8 and 9 are consistent with the outflow picture. In particular, the Bullet is clearly escaping from the gravitational potential of the Galactic center region even when the mass of the stellar cluster is included. In this model, however, it is not expected to see a tail produced behind the fast moving Blobs. The existence of a bow shock or X-ray emitting gas associated with the head of the Bullet would favor a model in which these fast-moving features are ejected by mass-losing stellar sources. Further observations of the Bullet are needed to understand its origin.

The comparison between the measured total velocities and the upper limits to the escape velocities at projected distance (r) from the center of each box to Sgr A* (Table 1) suggests that the ionized flow is on an unbound orbit around the Galactic center. This is consistent with the model of RYG that the ionized gas in the Northern Arm is on a hyperbolic orbit. Like the compact and relatively isolated features with high transverse velocities discussed above (e.g. the Bullet and the Blobs), the rest of the orbiting ionized features are extended and follow a global velocity field which may also be unbound to the Galactic center. If we use the projected distance as $\cos ^{-1}\left(45^{\circ}\right)$ of the actual distance, almost all the measured velocities are greater than the escape velocities listed in Table 1. The mass within the inner $10-20^{\prime \prime}$ is assumed to be dominated by a compact source centered 
on Sgr A* having a mass of $2.5 \times 10^{6} M_{\odot}$ as measured recently from stellar proper motion measurements (Eckart \& Genzel 1997). The escape velocity estimates may not be applicable at large distances where the mass of the evolved stellar cluster becomes important. From the comparison of the three dimensional stellar and ionized gas motions, it appears that these two systems are not dynamically coupled in the inner $20^{\prime \prime}$ of the Galactic center.

The effect of a strong gravitational potential due to the large concentration of dark matter near Sgr $\mathrm{A}^{*}$ is manifested as high velocity gradients of over $600 \mathrm{~km} \mathrm{~s}^{-1} \mathrm{pc}^{-1}$. However, the existence of ionized gas in an unbound orbit is inconsistent with the notion that the ionized gas in the Northern Arm is a tidally stretched infalling feature (e.g. Serabyn et al. 1988). Additionally, the present proper motion data do not support the interpretation that the Northern Arm is a segment of a one-armed spiral pattern induced as a result of an instability in the rotating disk (e.g. Lacy et al. 1991). A significant variation in the velocity distribution of ionized gas along Northern Arm is not consistent with a small variations expected from Keplerian motion, thus supporting that the Northern Arm is a material feature. We believe that the high velocity of ionized gas on an unbound orbit supports a scenario in which an energetic phenomenon outside the inner few parsecs of the Galactic center accelerated a cloud to pass by the Galactic center, which then collides with the CND and results in the loss of angular momentum of the material in the CND (Serabyn et al. 1988). There is evidence of disturbed neutral gas and shocked molecular gas based on $\mathrm{OH} 1720 \mathrm{MHz}$ maser emission at the interface of the extension of the Northern and Eastern Arms of Sgr A West and a "gap" in the CND (Yusef-Zadeh et al. 1996; Jackson et al. 1993). In this picture, the Northern and Eastern Arms delineate the edges of the intruding cloud photoionized by the UV radiation field at the Galactic center (Jackson et al. 1993). The neutral gas in the "gap" of the CND is interpreted to be the site of collision with a cloudlet pushed into the Galactic center, possibly by the energetic explosion of Sgr A East. Future modeling of the three-dimensional motion of ionized gas should constrain 
the inclination of the orbital plane of the ionized gas with respect to the orbital plane of the CND and examine the possibility that the CND is origin of the Northern and Eastern Arms.

F. Yusef-Zadeh's work was supported in part by NASA grant NAGW-2518. D. Roberts acknowledges support from the NSF grant AST94-19227. We thank Mark Wardle for useful discussion. 


\section{REFERENCES}

Backer, D.C. 1996, in IAU Symposium 169 eds: L. Blitz \& P. Teuben, p193

Biretta, J., Owen, F., \& Cornwell, T. 1989, ApJ, 342, 128

Biretta, J., Zhou, F. \& Owen, F. 1995, ApJ, 447, 582

Eckart, A. \& Genzel, R. 1997, MNRAS, 284, 576

Eckart, A. \& Genzel, R. 1996, Nature, 383, 415

Ekers, R. D., van Gorkom, J. H., Schwartz, U. J. \& Goss, W. M. 1983, AA, 122, 143

Güsten, R., Genzel, R., Wright, M.C.H., Jaffe, D.T., Stutzki, J. \& Harris,A.I., 1987, ApJ, 318,124

Herbst, T.M., Beckwith, S.V.M., Forrest, W.J. \& Pipher, J.L. 1993, AJ, 105, 956

Jackson, J. M., Geis, N., Genzel, R., Harris, A. I., Madden, S., Poglitsch, A., Stacey, G. J. \& Townes, C. H. 199, ApJ, 402, 173

Lacy, J.H., Achtermann, J.M.\& Serabyn, E. 1991, ApJ, 380, L71

Lutz, D., Krabbe, A., \& Genzel, R. 1993, ApJ, 418, 244

Roberts, D. \& Goss, W.M. 1993, ApJS, 86, 133

Roberts, D., Yusef-Zadeh, F. \& Goss, W.M. 1996, ApJ, 459, 627

Serabyn, E., Lacy, J.H., Townes, C.H., Bharat, R. 1988, ApJ, 326, 171

Wardle, M. \& Yusef-Zadeh, F., 1992, Nature, 357, 308

Yusef-Zadeh, F., Morris, M. \& Ekers, R.D. 1990, Nature, 348, 45

Yusef-Zadeh, F., Roberts, D.A., Goss, W.M., Frail, D. \& Green, A. 1996, ApJ, 466, L25

Zhao, J.-H., Goss, W.M., Lo, K.Y. \& Ekers, R.D. 1991, Nature, 354, 46 


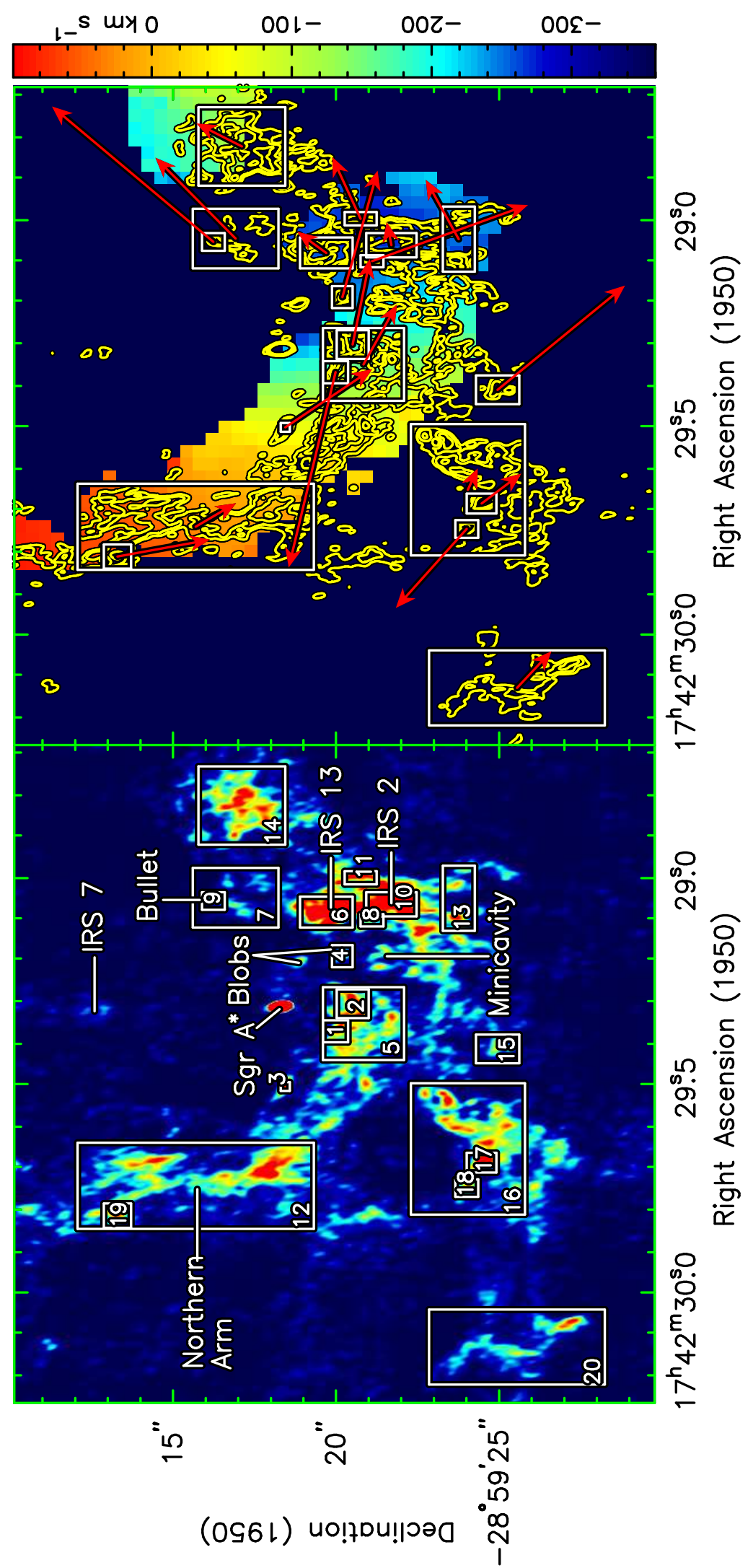

Fig. 1. - (a) A pseudo-color image of the $\lambda 2 \mathrm{~cm}$ continuum from the 1990 epoch data (left) with a resolution of $0{ }^{\prime \prime} 1 \times 00^{\prime \prime} 2$ and an $\mathrm{rms}$ noise of $0.104 \mathrm{mJy}_{\text {beam }}{ }^{-1}$. The boxes (see Table 1 ) are regions where proper motion measurements have been carried out with a $>3 \sigma$ level detection in at least one direction except Box 21. (b) The H92 $\alpha$ velocity distribution with spectral and spatial resolutions of $14 \mathrm{kms}^{-1}$ and $0.75 \times 1^{\prime \prime} .2$ presented in pseudo-color (right) with contours of the $\lambda 2 \mathrm{~cm}$ continuum image superposed. The length and direction of the vectors represent the transverse velocity in each box. The velocity and uncertainty of each vectors are presented in columns 6 and 7 of Table 1 . 


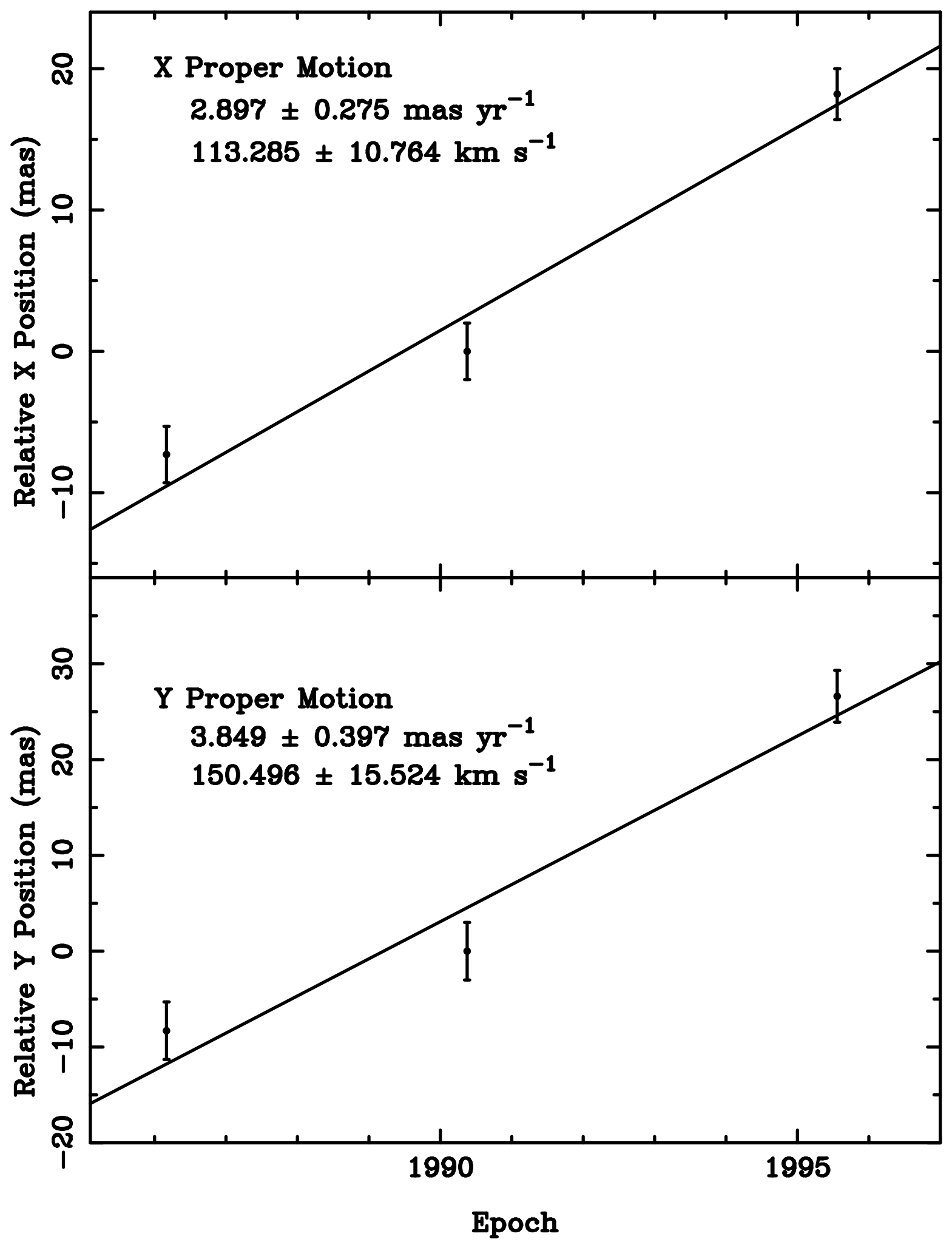

Fig. 2.- The change in $\alpha$ and $\delta$ as a function of three epochs for the region surrounding the IRS 13 cluster (Box 6 in Table 1). 


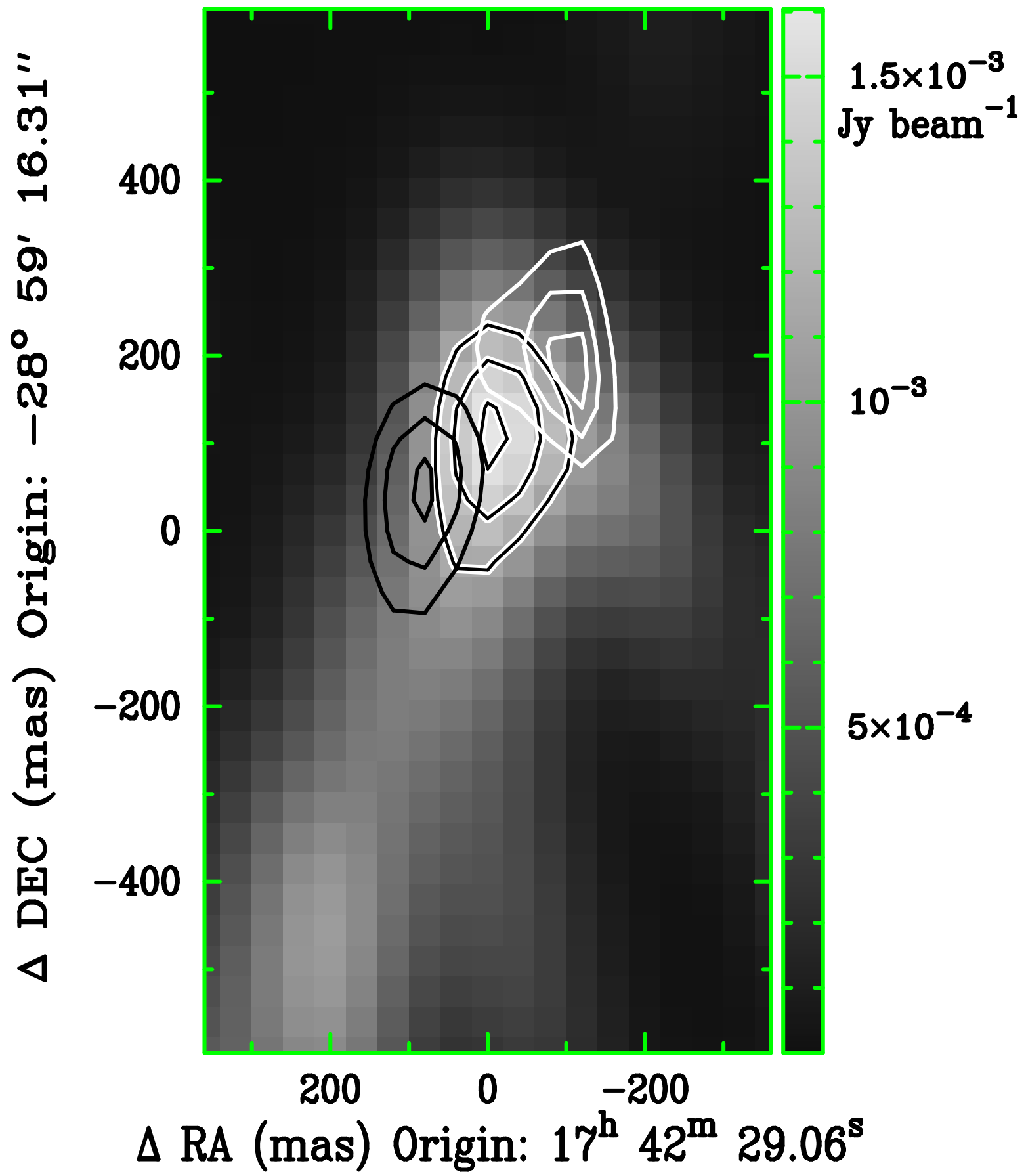

Fig. 3.- The contours of the peak continuum emission from the Bullet corresponding to three different epochs are superposed on the grayscale image of the source (including the tail) from the 1990 epoch observations. 
TABLE 1

Velocities of gas at the Galactic Center

\begin{tabular}{lccccccccc}
\hline \hline Box No. & $\begin{array}{c}\mathrm{r}^{a} \\
(\operatorname{arcsec})\end{array}$ & $\begin{array}{c}\mathrm{x}^{a} \\
\text { (arcsec) }\end{array}$ & $\begin{array}{c}\mathrm{y}^{a} \\
\text { (arcsec) }\end{array}$ & $\begin{array}{c}\text { Size }(\Delta \mathrm{x} \times \Delta \mathrm{y}) \\
\text { (asec } \times \text { a sec })\end{array}$ & $\begin{array}{c}V_{\mathrm{x}} \\
\left(\mathrm{kms}^{-1}\right)\end{array}$ & $\begin{array}{c}V_{\mathrm{y}} \\
\left(\mathrm{kms}^{-1}\right)\end{array}$ & $\begin{array}{c}V_{\mathrm{z}} \\
\left(\mathrm{kms}^{-1}\right)\end{array}$ & $\begin{array}{c}V_{\text {tot }} \\
\left(\mathrm{kms}^{-1}\right)\end{array}$ & $\begin{array}{c}V_{\text {esc }} \\
\left(\mathrm{km} \mathrm{s}^{-1}\right)\end{array}$ \\
\hline 1 & 1.834 & +0.735 & -1.68 & $0.7 \times 0.77$ & $-1041 \pm 315$ & $241 \pm 230$ & -184 & $1085 \pm 306$ & 549 \\
2 & 2.21 & -.14 & -2.2 & $0.91 \times 0.98$ & $+456 \pm 97$ & $-89 \pm 89$ & -222 & $515 \pm 87$ & 500 \\
4 (Blob $\zeta)$ & 2.49 & -2.48 & -0.14 & $0.28 \times 0.28$ & $+310 \pm 131$ & $-447 \pm 122$ & -76.8 & $544 \pm 125$ & 471 \\
5 & 2.51 & -1.645 & -1.89 & $0.7 \times 0.63$ & $+674 \pm 128$ & $-182 \pm 323$ & - & $698 \pm 140$ & 469 \\
6 (IRS 13) & 2.58 & +0.49 & -2.53 & $2.31 \times 2.48$ & $+319 \pm 55$ & $-172 \pm 41$ & -177 & $404 \pm 47$ & 463 \\
7 & 3.35 & -3.04 & -1.4 & $0.98 \times 1.61$ & $+113 \pm 11$ & $+150 \pm 15$ & -177 & $258 \pm 10$ & 406 \\
8 & 3.76 & -3.5 & +1.38 & $1.89 \times 2.62$ & $+431 \pm 118$ & $+413 \pm 208$ & -35 & $598 \pm 167$ & 384 \\
9 (Bullet) & 3.94 & +2.78 & -2.8 & $0.38 \times 0.7$ & $+300 \pm 217$ & $-804 \pm 255$ & -259.2 & $896 \pm 240$ & 375 \\
10 (IRS 2) & 3.97 & -3.39 & +2.065 & $0.56 \times 0.7$ & $+722 \pm 156$ & $+832 \pm 203$ & - & $1102 \pm 184$ & 373 \\
11 & 4.73 & -3.29 & -3.39 & $0.77 \times 1.54$ & $+122 \pm 11$ & $+24 \pm 34$ & -270 & $297 \pm 5.4$ & 342 \\
12 (N. Arm) & 6.2 & -5.13 & -2.45 & $0.42 \times 0.98$ & $+329 \pm 56$ & $+146 \pm 143$ & -273 & $452 \pm 62$ & 339 \\
13 & 6.47 & -3.48 & -5.46 & $2.73 \times 7.24$ & $+126 \pm 30$ & $-207 \pm 58$ & +29.9 & $244 \pm 51$ & 299 \\
14 (IRS 6) & 6.53 & -6.42 & +1.19 & $2.46 \times 2.06$ & $+316 \pm 16$ & $+170 \pm 73$ & -259 & $442 \pm 49$ & 292 \\
15 & 6.77 & -1.29 & -6.65 & $0.91 \times 1.33$ & $+556 \pm 236$ & $-657 \pm 201$ & - & $861 \pm 216$ & 286 \\
16 (IRS 9) & 7.27 & +4.46 & -5.74 & $4.16 \times 3.5$ & $+114 \pm 36$ & $-51 \pm 52$ & $+130^{b}$ & $180 \pm 27$ & 276 \\
17 & 7.87 & +4.9 & -6.16 & $0.63 \times 0.91$ & $+160 \pm 46$ & $-205 \pm 80$ & +154 & $303 \pm 59$ & 265 \\
18 & 8.07 & +5.7 & -5.7 & $0.56 \times 0.7$ & $-421 \pm 142$ & $+366 \pm 152$ & $+147^{b}$ & $577 \pm 142$ & 262 \\
19 & 8.267 & +6.58 & +5 & $0.77 \times 0.84$ & $+90 \pm 89$ & $-477 \pm 95$ & +66 & $490 \pm 94$ & 259 \\
20 (IRS 4) & 12.96 & +10.74 & -7.24 & $2.38 \times 5.39$ & $+196 \pm 59$ & $-178 \pm 137$ & $+171^{b}$ & $315 \pm 86$ & 207 \\
21 (IRS 1) & 5.27 & -5.25 & +0.42 & $0.77 \times 0.91$ & $+25 \pm 48$ & $-172 \pm 63$ & - & $174 \pm 62$ & 324 \\
22 (IRS 8) & 29.44 & +1.54 & +29.4 & $0.77 \times 0.77$ & $-54 \pm 73$ & $+97 \pm 83$ & - & $111 \pm 81$ & 137 \\
\hline
\end{tabular}

${ }^{a}$ position relative to $\mathrm{Sgr} \mathrm{A}^{*}$.

${ }^{b}$ Radial velocity from Roberts \& Goss (1993), all others are from RYG. 